\title{
Nonlinear optics with stationary pulses of light
}

\author{
A. André, M. Bajcsy, A. S. Zibrov, and M. D. Lukin \\ Physics Department, Harvard University, Cambridge, MA 02138
}

(Dated: October 16, 2018)

\begin{abstract}
We show that the recently demonstrated technique for generating stationary pulses of light [Nature 426, 638 (2003)] can be extended to localize optical pulses in all three spatial dimensions in a resonant atomic medium. This method can be used to dramatically enhance the nonlinear interaction between weak optical pulses. In particular, we show that an efficient Kerr-like interaction between two pulses can be implemented as a sequence of several purely linear optical processes. The resulting process may enable coherent interactions between single photon pulses.
\end{abstract}

Techniques that could facilitate controlled nonlinear interactions between few-photon light pulses are now actively explored [1]. Although research into fundamental limits of nonlinear optics has been carried out over the last three decades, there is renewed interest in these problems in part due to e.g. potential applications in quantum information science [2]. In general, such interactions between few-photon pulses are difficult to achieve, as they require a combination of large nonlinearity, low photon loss and tight confinement of the light beams [3]. In addition, long atom-photon interaction times are required. Simultaneous implementation of all of these requirements is by now only feasible in the context of cavity QED [4]

In this Letter we describe a novel method for achieving nonlinear interaction between weak light pulses. Our method is based on a recently demonstrated technique [5, 6] in which light propagating in a medium of $\mathrm{Rb}$ atoms was converted into an excitation with localized, stationary electromagnetic energy, which could be held and released after a controllable interval. This is achieved by using Electromagnetically Induced Transparency (EIT) 7] to coherently control the pulse propagation. We show here that this method can be extended to confine stationary pulses in all three spatial dimensions. This, in turn, can be used to strongly enhance the nonlinear interaction between weak pulses of light. Specifically we demonstrate that an efficient Kerr-like interaction between two pulses can be implemented as a sequence of linear optical processes and atomic state manipulations. Coherent, controlled nonlinear processes at optical energies corresponding to a single light quanta appear feasible.

Before proceeding, we note that the present work is closely related to recent studies on the resonant enhancement of nonlinear optical phenomena via EIT 8, 9, 10, 11]. The essence of these studies is to utilize steep atomic dispersion associated with narrow EIT resonances. In such a system, a small AC Stark shift associated with a weak off-resonant pulse of signal light, produces a large change in refractive index for a resonant probe pulse. In order to fully take advantage of this process, long interaction times between signal and probe pulses must be ensured. Although the latter can be achieved by reducing the group velocities of two interacting pulses by equal amounts [12], in practice this results only in a modest increase of the nonlinear optical efficiency since reduction of the group velocity is accompanied by a corresponding decrease of the light energy in the propagating pulse. Moreover, such nonlinear interaction is accompanied by pulse distortion, which poses a fundamental limit to nonlinear interactions. In contrast, the technique presented here allows for long interaction times (associated with stationary light pulses) without proportional reduction of the photon energy. Furthermore, our technique enables light localization in all three spatial dimensions in the presence of atoms, and allows to entirely avoid competing effects such as pulse distortion.

The ideas discussed in Refs. 6] allow one to localize and hold a pulse of light within a stationary envelope along the propagation direction. In practice, focused pulses will undergo diffraction in the transverse directions. In order to prevent diffraction, it is necessary to confine the signal beam in transverse directions. This can be achieved, e.g. by using a hollow core photonic crystal fiber filled with an active medium of resonant atoms [13]. Such an approach is particularly attractive in that it ensures a single-mode beam quality for interacting beams. The unwanted interactions of atoms with fiber walls can be avoided by using atom guiding techniques [14]. Alternatively, the signal pulse guiding can be accomplished by using focused control beams. Shaped control beams can be used to create a transverse variation of the index of refraction, enabling waveguiding and confinement of light pulses to small transverse dimensions.

To be specific we consider a medium of length $L$ consisting of an ensemble of $N$ three-level atoms in the $\Lambda$ configuration, with two metastable lower states, as shown in Fig. 1a. The ground states $|g\rangle,|s\rangle$ are coupled to the excited state $|e\rangle$ via a control field applied on resonance with the $|s\rangle \rightarrow|e\rangle$ transition and a weak quantized signal field close to resonance with the $|g\rangle \rightarrow|e\rangle$ transition. The control field consists of two counterpropagating fields with spatially and temporally varying Rabi frequencies $\Omega_{ \pm}(\vec{r}, t)$, so that the control field Rabi frequency is $\Omega(\vec{r}, t)=\Omega_{+}(\vec{r}, t) e^{i k_{c} z}+\Omega_{-}(\vec{r}, t) e^{-i k_{c} z}$, where $k_{c}=n_{c} \omega_{e s} / c, n_{c}$ being the background index of refraction at the frequency $\omega_{e s}$. The corresponding signal fields have slowly varying envelopes $\hat{E}_{ \pm}(\vec{r}, t)$, so that 
a.

b.
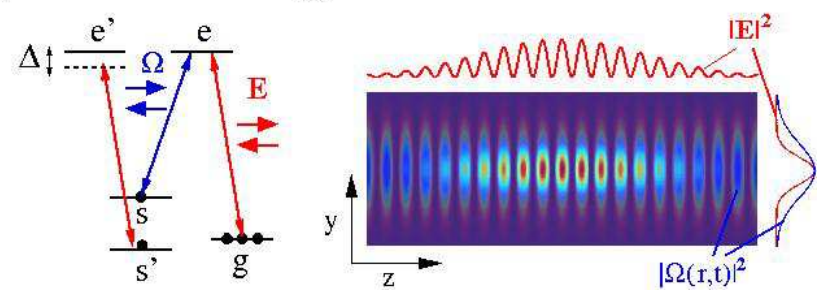

FIG. 1: a. Three-level atoms in $\Lambda$-configuration, with auxiliary levels $\left|s^{\prime}\right\rangle,\left|e^{\prime}\right\rangle$. Forward and backward propagating control fields with Rabi frequencies $\Omega_{ \pm}$, and weak signal field $\mathcal{E}_{ \pm}$. b. Three-dimensional confinement and waveguiding of signal light due to transverse intensity profile and longitudinal modulation of control field.

the signal field is

$$
\begin{aligned}
\hat{E}_{S}^{(+)}(\vec{r}, t) & =\left(\frac{\hbar \omega_{0}}{2 \epsilon_{0} V}\right)^{1 / 2}\left[\hat{E}_{+}(\vec{r}, t) e^{i k_{s} z}\right. \\
& \left.+\hat{E}_{-}(\vec{r}, t) e^{-i k_{s} z}\right] e^{-i \omega_{e g} t},
\end{aligned}
$$

where $k_{s}=n_{s} \omega_{e g} / c, V$ is the quantization volume, and $n_{s}$ is the background refractive index at the frequency $\omega_{e g}$ due to off-resonant atomic levels. Note that in practice $n_{s}$ can be tuned, e.g. by changing the light polarization.

We describe the atomic properties with slowly varying collective operators [15] $\hat{\sigma}_{\mu \nu}(\vec{r}, t)=$ $\frac{1}{N_{\vec{r}}} \sum_{j=1}^{N_{\vec{r}}}|\mu\rangle_{j}\langle\nu| e^{-i \omega_{\mu \nu} t}$ where $\omega_{\mu \nu}=\left(E_{\mu}-E_{\nu}\right) / \hbar$, and where $N_{\vec{r}}$ is the number of atoms in a small but macroscopic volume around position $\vec{r}$. We define the polarization operator to be $\hat{P}(\vec{r}, t)=\sqrt{N} \hat{\sigma}_{g e}(\vec{r}, t)$, and the spin flip operator $\hat{S}(\vec{r}, t)=\sqrt{N} \hat{\sigma}_{g s}(\vec{r}, t)$. In the present situation of weak signal fields and strong control fields, most atoms are in state $|g\rangle$, with a few spin-flipped atoms in $|s\rangle$, so that the polarization and spin flip operators obey bosonic commutation relations [15]. Associated with the forward/backward propagating fields are slowly varying polarization envelopes $\hat{P}_{ \pm}(\vec{r}, t)$, so that the total polarization is $\hat{P}(\vec{r}, t)=\hat{P}_{+}(\vec{r}, t) e^{i k_{s} z}+\hat{P}_{-}(\vec{r}, t) e^{-i k_{s} z}$.

Letting the wavevector mismatch between copropagating signal and control fields be $\Delta K=k_{s}-k_{c}=$ $\left(n_{s} \omega_{e g}-n_{c} \omega_{e s}\right) / c$, and defining $\hat{\mathcal{E}}_{ \pm}=\hat{E}_{ \pm} e^{ \pm i \Delta K z}$ and $\hat{\mathcal{P}}_{ \pm}=\hat{P}_{ \pm} e^{ \pm i \Delta K z}$, the Heisenberg equations of motion for the slowly varying operators $\hat{\mathcal{E}}_{ \pm}(\vec{r}, t)[15]$ can be written as (in the paraxial approximation)

$$
\left(\frac{\partial}{\partial t} \pm c \frac{\partial}{\partial z}-i \frac{c \nabla_{T}^{2}}{2 k_{s}}\right) \hat{\mathcal{E}}_{ \pm}=i \Delta K c \hat{\mathcal{E}}_{ \pm}+i g \sqrt{N} \hat{\mathcal{P}}_{ \pm}
$$

where $\nabla_{T}^{2}=\nabla^{2}-\frac{d^{2}}{d z^{2}}$ is the transverse Laplacian, and where $g=\wp\left(\frac{\omega_{0}}{2 \hbar \epsilon_{0} V}\right)^{1 / 2}$ is the atom-field coupling constant, $\wp$ being the dipole matrix element for the $|g\rangle-|e\rangle$ transition, and $V$ the quantization volume.

Following [5, 15] we introduce two components $\hat{\Psi}_{ \pm}$of a coupled excitation of light and an atomic spin wave ("dark-state polariton") corresponding to forward and backward signal fields respectively. In the experimentally relevant case of small group velocities [16, 17] the polariton components are represented by $\hat{\Psi}_{ \pm}=g \sqrt{N} \hat{\mathcal{E}}_{ \pm} / \Omega_{ \pm}$. In the adiabatic limit of slowly varying pulses, disregarding the slow decay of ground state coherence, and Fourier transforming $\left(\partial_{t} \rightarrow-i \omega\right)$, we find

$$
\begin{gathered}
\left(c \frac{\partial}{\partial z}-i \frac{c \nabla_{T}^{2}}{2 k_{0}}\right) \hat{\Psi}_{+}=i \Delta K c \hat{\Psi}_{+} \\
+i \eta \omega\left(\alpha_{+} \hat{\Psi}_{+}+\alpha_{-} \hat{\Psi}_{-}\right)-\alpha_{-} \xi\left(\hat{\Psi}_{+}-\hat{\Psi}_{-}\right)+\hat{F}_{+}(\vec{r}, \omega) \\
\quad\left(-c \frac{\partial}{\partial z}-i \frac{c \nabla_{T}^{2}}{2 k_{0}}\right) \hat{\Psi}_{-}=i \Delta K c \hat{\Psi}_{-} \\
+i \eta \omega\left(\alpha_{+} \hat{\Psi}_{+}+\alpha_{-} \hat{\Psi}_{-}\right)+\alpha_{+} \xi\left(\hat{\Psi}_{+}-\hat{\Psi}_{-}\right)+\hat{F}_{-}(\vec{r}, \omega)
\end{gathered}
$$

where $k_{0}=\omega_{e g} / c, \eta=\frac{g^{2} N}{\left|\Omega_{+}\right|^{2}+\left.\Omega_{-}\right|^{2}}, \alpha_{ \pm}=\frac{\left|\Omega_{+}\right|^{2}}{\left|\Omega_{+}\right|^{2}+\left.\Omega_{-}\right|^{2}}$, and $\xi=\frac{g^{2} N}{\gamma}$. We have also assumed that $k_{0} a \gg 1$, where $a$ is the typical transverse size of the control beams. These equations describe two slow waves that are coupled due to periodic modulation of atomic absorption and group velocity. The terms containing $\xi$ on the right hand side of Eqns. (3) are proportional to the absorption coefficient $\xi$ near resonant line center. $\hat{F}_{ \pm}(\vec{r}, \omega)$ are noise forces associated with dissipation. When $\xi$ is large these terms give rise to the pulse matching phenomenon [7]: whenever one of the fields is created the other will adjust itself within a short propagation distance to match its amplitude such that $\hat{\Psi}_{+}-\hat{\Psi}_{-} \rightarrow 0[\underline{6}]$.

In order to achieve transverse confinement of light pulses, we take into account the transverse dependence of the control field intensity and the resulting variation of the index of refraction. For a focused control beam, the intensity decreases with distance from the optical axis, so that for negative two-photon detuning (as is necessary for phasematching), the index of refraction decreases with distance from the optical axis. This leads to waveguiding of the signal light. The combination of waveguiding with strong coupling of forward and backward propagating modes, permits the complete three-dimensional confinement of light pulses in the medium.

We assume a transverse spatial variation of the control field intensity, e.g. for a weakly focused gaussian beam, $\left|\Omega_{ \pm}(r)\right|^{2}=\left|\Omega_{ \pm}(0)\right|^{2} e^{-(r / a)^{2}}$. Expanding for $r \ll a$, we have $\eta(r)=\eta_{0}\left[1+(r / a)^{2}+\cdots\right]$. We consider trial solutions of (3) of the from $\Psi_{+}=A_{+} e^{i \beta z-(r / R)^{2}}$ and $\Psi_{-}=A_{-} e^{-i \beta z-(r / R)^{2}}$ where $\beta, R$ and $A_{+} / A_{-}$are determined by requiring that the coefficients of different powers of $r$ vanish independently. With these requirements we find the two solutions $R=\infty$ and

$$
R=\left(-\frac{2 a^{2} c}{k_{0} \eta \omega}\right)^{1 / 4} .
$$


The eigenvector of the finite solution has $A_{+} / A_{-}=1$, which corresponds to the stationary pulses [6] for which $\Psi_{+}-\Psi_{-} \rightarrow 0$. We also find the dispersion relation

$$
\eta \omega=\left(\frac{2 c}{k_{0} R(\omega)^{2}}-\Delta K c\right)-i \frac{(c \beta)^{2}}{\xi}+\left(\alpha_{+}-\alpha_{-}\right) c \beta .
$$

In the time-domain this corresponds to propagation at a group velocity $v_{g}=c \frac{\alpha_{+}-\alpha_{-}}{\eta}$, that can be controlled by adjusting the intensities $I_{ \pm}(t) \propto\left|\Omega_{ \pm}(t)\right|^{2}$ of the counterpropagating control fields. Due to the imaginary term, there is also a slow spreading of the stationary pulse at a rate $\delta l / l \sim \sqrt{c^{2} t /\left(\eta \xi l^{2}\right)}$, which determines the maximal trapping time of the stationary excitation.

We are interested in a simultaneous solution of Eqs.(3) when $\alpha_{+} \approx \alpha_{-}$and the optical depth $\xi$ is large. This yields the radius of the stationary pulse $R=$ $a 2^{1 / 4}\left[\sqrt{1+\Delta K k_{0} a^{2}}-1\right]^{-1 / 2}$, which under conditions of strong confinements $\Delta K k_{0} a^{2} \gg 1$ results in

$$
R=a\left[2 /\left(\Delta K k_{0} a^{2}\right)\right]^{1 / 4} .
$$

Hence, in an optically dense medium $(\xi L / c \gg 1)$ a stationary excitation confined in all three dimensions can be controllably created. To be specific, for atomic Rb $(\lambda=0.8 \mu \mathrm{m})$ at density $n=10^{14} \mathrm{~cm}^{-3}$, we take the background refractive index due to off-resonant levels to be $n-1=1.2 \cdot 10^{-2}$ [18. For a Gaussian control beam with waist $a \sim 100 \mu \mathrm{m}$ at the center of the atomic cell ( the corresponding Rayleigh range is $z_{0}=3.9 \mathrm{~cm}$ ), we find that the guided mode radius is $R=13 \mu \mathrm{m}$ (for which the Rayleigh range is $z_{0}=0.06 \mathrm{~cm}$ ), so that the diffraction-free range is extended by a factor of 60 . When the control beam is chosen in the form of a non-diffracting Bessel beam [19], waveguiding over much longer propagation distances is possible. For example, with a beam in which the radius of the first lobe of the Bessel function is $a=20 \mu \mathrm{m}$, the guided mode radius is $R \sim 5.7 \mu \mathrm{m}$. In practice, this allows confinement over tens of $\mathrm{cm}$, whereas in free space the corresponding Rayleigh range would only be $0.01 \mathrm{~cm}$. Reducing the control beam radius until $R \sim a$, gives an estimate of the smallest guided mode achievable, which for an index of $n-1=1.2 \cdot 10^{-2}$ is $R_{\text {min }}=1.6 \mu \mathrm{m}$.

We next turn to the nonlinear optical interaction between two weak pulses of light. A notable feature of the step by step process described below, is that it consists of a sequence of purely linear optical interactions and atomic state manipulations, leading to an effective optical nonlinear interaction. As shown in the timing diagram Fig. 2a, a light pulse (signal pulse) travelling through the atomic medium is initially stored in the $|g\rangle\langle s|$ coherence. Next, a Raman or microwave $\pi$ pulse (RA) transfers the population from $|s\rangle$ to $\left|s^{\prime}\right\rangle$ (see Fig. 2a), thereby transferring the stored excitation to the spin excitation $\hat{\mathcal{S}}^{\prime}=\sqrt{N} \hat{\sigma}_{g s^{\prime}}$. The signal pulse is stored as a spin wave $\sigma_{g s^{\prime}}(\vec{r}, t)=U_{s}(\vec{\rho}) \hat{\mathcal{S}}^{\prime}(z, t) / \sqrt{N}$, where $U_{s}(\vec{\rho})$ describes the transverse spin wave mode.

A second light pulse (probe pulse) is then sent through the medium and stored in the coherence $\hat{\mathcal{S}}$. The spin excitation associated with the probe pulse is then converted into a stationary excitation. The latter is then moved through the stored spin excitation associated with the signal pulse. Probe light in the modes $\hat{\mathcal{E}}_{ \pm}$interacts dispersively with atoms in level $\left|s^{\prime}\right\rangle$ (see Fig. 1a), thereby acquiring a phase shift proportional to the number of excitations $\hat{\mathcal{S}}^{\prime} \hat{\mathcal{S}}^{\prime}$, leading to an effective Kerr-type nonlinearity.

We focus on the situation where the transverse spatial size of the signal spin wave $\hat{\mathcal{S}}^{\prime}$ is much smaller than the probe transverse size. Under these assumptions and writing the guided mode transverse dependence as $\hat{\Psi}_{ \pm}(r, z, t)=e^{-(r / R)^{2}} \hat{\psi}_{ \pm}(z, t)$, we find, in terms of the polariton components $\hat{\psi}_{ \pm}$,

$$
\begin{aligned}
\left(\partial_{t}+c \partial_{z}\right) \hat{\psi}_{+} & =-\eta \partial_{t}\left(\alpha_{+} \hat{\psi}_{+}+\alpha_{-} \hat{\psi}_{-}\right)-\alpha_{-} \xi\left(\hat{\psi}_{+}-\hat{\psi}_{-}\right) \\
& +i \beta \hat{\mathcal{S}}^{\dagger} \hat{\mathcal{S}}^{\prime} \hat{\psi}_{+}+\hat{F}_{+}(z, t) \\
\left(\partial_{t}-c \partial_{z}\right) \hat{\psi}_{-} & =-\eta \partial_{t}\left(\alpha_{+} \hat{\psi}_{+}+\alpha_{-} \hat{\psi}_{-}\right)+\alpha_{+} \xi\left(\hat{\psi}_{+}-\hat{\psi}_{-}\right) \\
& +i \beta \hat{\mathcal{S}}^{\dagger} \hat{\mathcal{S}}^{\prime} \hat{\psi}_{-}+\hat{F}_{-}(z, t)
\end{aligned}
$$

where $\beta=\frac{\tilde{g}^{2}}{\Delta}(1+i \gamma / \Delta)$, with $\tilde{g}=g \frac{A}{\pi R^{2}}, A$ is the quantization area, and $\Delta$ is the detuning of the fields $\hat{\mathcal{E}}_{ \pm}$from the optical transition $\left|s^{\prime}\right\rangle \rightarrow\left|e^{\prime}\right\rangle$ (see Fig. 2a). The interaction of the localized excitation $\hat{\mathcal{S}}^{\prime}$ with the guided modes $\hat{\psi}_{ \pm}$does not depend on the transverse coordinate, and the effective transverse area corresponds to the mode area $\pi R^{2}$, in complete analogy to the interaction of localized atoms with the field mode in cavity QED [4].

In the stationary pulse configuration [6], the stationary excitation is bound to the spin wave and $\hat{\mathcal{S}} \simeq$ $-\left(\alpha_{+} \hat{\psi}_{+}+\alpha_{-} \hat{\psi}_{-}\right)$. Solving adiabaticaly, in the limit of large optical depth $\xi$ and writing $v_{g}=\left(\alpha_{+}-\alpha_{-}\right) c / \eta$, we find

$$
\begin{aligned}
{\left[\partial_{t}+v_{g} \partial_{z}\right] \hat{\mathcal{S}} } & =i \frac{\beta}{\eta}\left[\hat{\mathcal{S}}^{\dagger} \hat{\mathcal{S}}^{\prime}\right] \hat{\mathcal{S}}+\left[4 \alpha_{+} \alpha_{-} \frac{\left(c \partial_{z}\right)^{2}}{\eta \xi}\right] \hat{\mathcal{S}} \\
\partial_{t} \hat{\mathcal{S}}^{\prime} & =i \frac{\beta}{\eta}\left[\hat{\mathcal{S}}^{\dagger} \hat{\mathcal{S}}\right] \hat{\mathcal{S}}^{\prime}
\end{aligned}
$$

where we have ignored for now absorption and the associated noise.

To solve for $\hat{\mathcal{S}}(z, t)$ and $\hat{\mathcal{S}}^{\prime}(z, t)$, we first ignore pulse spreading. Let $\hat{n}_{1}(z)=\hat{\mathcal{S}}^{\dagger} \hat{\mathcal{S}}^{\prime}$ (independent of $t$ ), and $\hat{n}_{2}(z, t)=\hat{\mathcal{S}}^{\dagger} \hat{\mathcal{S}}$ (which depends only on the variable $t^{\prime}=$ 


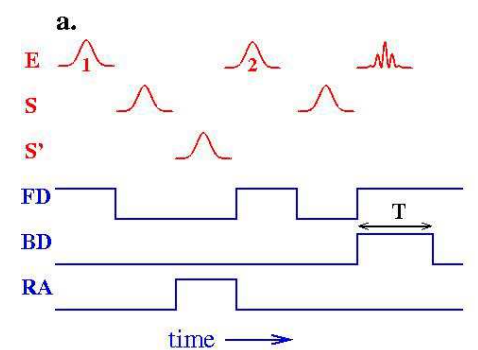

b.

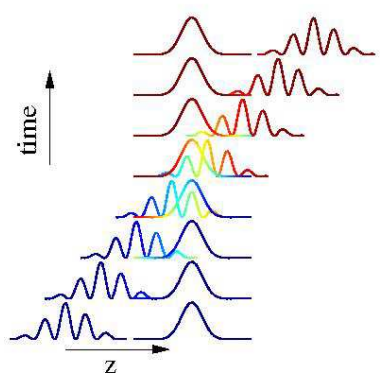

FIG. 2: Nonlinear optical interaction with weak pulses as sequence of linear operations. a. Timing diagram: forward (FD), backward (BD), and Raman or microwave (RA) intensities vs. time. b. Illustration of Kerr interaction between slowly propagating stationary pulse $\mathcal{S}$ and stored excitation $\mathcal{S}^{\prime}$ leading to phase shift (represented as changing color) of pulses.

$\left.t-z / v_{g}\right)$. We find

$$
\begin{aligned}
& \hat{\mathcal{S}}(z, t)=\exp \left[i \frac{\beta}{\eta v_{g}} \int_{z-v_{g} t}^{z} d z^{\prime} \hat{n}_{1}\left(z^{\prime}\right)\right] \hat{\mathcal{S}}\left(z-v_{g} t, 0\right) \\
& \hat{\mathcal{S}}^{\prime}(z, t)=\exp \left[i \frac{\beta}{\eta v_{g}} \int_{0}^{v_{g} t-z} d z^{\prime} \hat{n}_{2}\left(-z^{\prime}, 0\right)\right] \hat{\mathcal{S}}^{\prime}(z, 0)
\end{aligned}
$$

When the slowly moving pulse $\hat{\mathcal{S}}$ has completely traversed the stored spin coherence $\hat{\mathcal{S}}^{\prime}$, the phase shift is $\phi_{S}=\frac{\operatorname{Re}[\beta]}{\eta v_{g}} L \hat{N}_{\mathcal{S}^{\prime}}$ (where $\hat{N}_{\mathcal{S}^{\prime}}$ is the number of excitations initially stored in $\hat{\mathcal{S}}^{\prime}$ ). The phase shift is proportional to the interaction time, i.e. inversely proportional to the group velocity of the slowly moving pulse $\hat{\mathcal{S}}$. To estimate the maximal phase shift, we note that the group velocity must be large enough that $v_{g} t \gtrsim l_{s^{\prime}}$, where $l_{s^{\prime}}$ is the length of spin coherence envelope. Also, non-adiabatic corrections due to the pulse spreading term in (8a) should be small, so that $\frac{\left(c / l_{S}\right)^{2}}{\eta \xi} t \lesssim 1$. Putting these two conditions together yields

$$
\phi_{S} \lesssim d_{0} \frac{\gamma}{\Delta} \frac{\sigma}{\pi R^{2}}\left(\frac{l_{s}^{2}}{L l_{s^{\prime}}}\right)
$$

where the resonant scattering cross-section is $\sigma=\frac{3}{4 \pi} \lambda^{2}$. Note that the nonlinear phase shift scales linearly with the optical depth $d_{0}$, in contrast to scaling with $\sqrt{d_{0}}$ for the case of two slowly propagating pulses [12], in which case pulse distortion effects are also significant.

Specifically, a $300 \mu \mathrm{m}$ long, cigar shaped cloud of cold ${ }^{87} \mathrm{Rb}$ atoms confined in an optical dipole trap at a density of $n \sim 10^{14} \mathrm{~cm}^{-3}$ has an optical depth in excess of $d_{0} \sim$ $10^{3}$. Similar optical depth can be potentially achieved by guiding cold atom clouds of smaller density in a photonic crystal fiber [13, 14]. Taking the guided mode radius $R \simeq 2 \mu \mathrm{m}$ and accounting for absorption losses, we choose the detuning to be $\Delta \simeq 16 \gamma$ and find that a phase shift of $\phi_{S} \sim \pi$ is achievable due to a single stored quantum. Under these conditions the two-photon loss probability is a few percent.

To summarize, we have shown that three-dimensional confinement of light pulses is possible by combining the technique of stationary light pulses with the transverse light guiding. This technique can be used to engineer efficient nonlinear optical interactions leading to significant phase shifts for weak optical pulses. Such interactions have interesting applications ranging from QND measurements [20] of few-photon pulses to quantum information processing [2].

We gratefully acknowledge discussions with S. Harris and G. Kurizki. This work was supported by DARPA, the NSF, Alfred P. Sloan and David and Lucille Packard Foundations.

[1] M. D. Lukin and A. Imamoğlu, Nature, 413, 273 (2001).

[2] The Physics of Quantum Information, edited by $\mathrm{D}$. Bouwmeester, A. Ekert, and A. Zeilinger (Springer, New York, 2000).

[3] R. W. Boyd, Nonlinear Optics, (Academic Press, San Diego, 2003).

[4] H. Mabuchi and A. C. Doherty, Science 298, 1372 (2002); J. M. Raimond, M. Brune, and S. Haroche, Rev. Mod. Phys.73, 565 (2001).

[5] A. André and M. D. Lukin, Phys. Rev. Lett. 89, 143602 (2002).

[6] M. Bajcsy, A. S. Zibrov, and M. D. Lukin, Nature 426, 638 (2003).

[7] S. E. Harris, Phys. Today 50, 36 (1997).

[8] H. Schmidt and A. Imamoğlu, Opt Lett. 21, 1936 (1996).

[9] S. E. Harris and Y. Yamamoto, Phys. Rev. Lett. 81, 3611 (1998); S. E. Harris and L. V. Hau, Phys. Rev. Lett. 82, 4611 (1999).

[10] D. Petrosyan and G. Kurizki, Phys. Rev. A 65, 033833 (2002).

[11] M. Mašalas and M. Fleischhauer, Phys. Rev. A 69, 061801(R) (2004).

[12] M. D. Lukin and A. Imamoğlu, Phys. Rev. Lett. 84, 1419 (2000).

[13] F. Benabid et al., Phys. Rev. Lett. 93, 123903 (2004); S. O. Konorov, A. B. Fedotov, and A. M. Zheltikov, Opt. Lett. 28, 1448 (2003).

[14] M. J. Renn et al., Phys. Rev. Lett. 75, 3253 (1995); D. Müller et al., Phys. Rev. A 61, 033411 (2000).

[15] M. Fleischhauer and M. D. Lukin, Phys. Rev. Lett. 84, 5094 (2000).

[16] L. V. Hau et al., Nature 397, 594 (1999); M. M. Kash et al., Phys. Rev. Lett. 82, 5229 (1999).

[17] C. Liu et al.. Nature 409, 490 (2001); D. F. Phillips et al., Phys. Rev. Lett. 86, 783 (2001).

[18] A. S. Zibrov et al., J. Mod. Opt. 49, 359 (2002).

[19] J. Durnin, J. Opt. Soc. Am. A 4, 651 (1987).

[20] Ph. Grangier, J.-A. Levenson, and J.-Ph. Poizat, Nature 396, 537 (1998). 